Supporting Information for:

\title{
Glass-like Lattice Thermal Conductivity and Thermoelectric Properties of Incommensurate Chimney-Ladder Compound $\mathrm{FeGe}_{\gamma}$
}

Naoki Sato, ${ }^{*, \dagger}$ Hideyasu Ouchi, ${ }^{\dagger}$ Yoshiki Takagiwa, ${ }^{\ddagger}$ and Kaoru Kimura ${ }^{\dagger}$

${ }^{\dagger}$ Department of Advanced Materials Science, The University of Tokyo, 5-1-5 Kashiwanoha, Kashiwa, Chiba 277-8561, Japan, ${ }^{\ddagger}$ National Institute for Materials Science, 1-2-1 Sengen, Tsukuba, Ibaraki 305-0047, Japan

*E-mail: nsato@phys.mm.t.u-tokyo.ac.jp
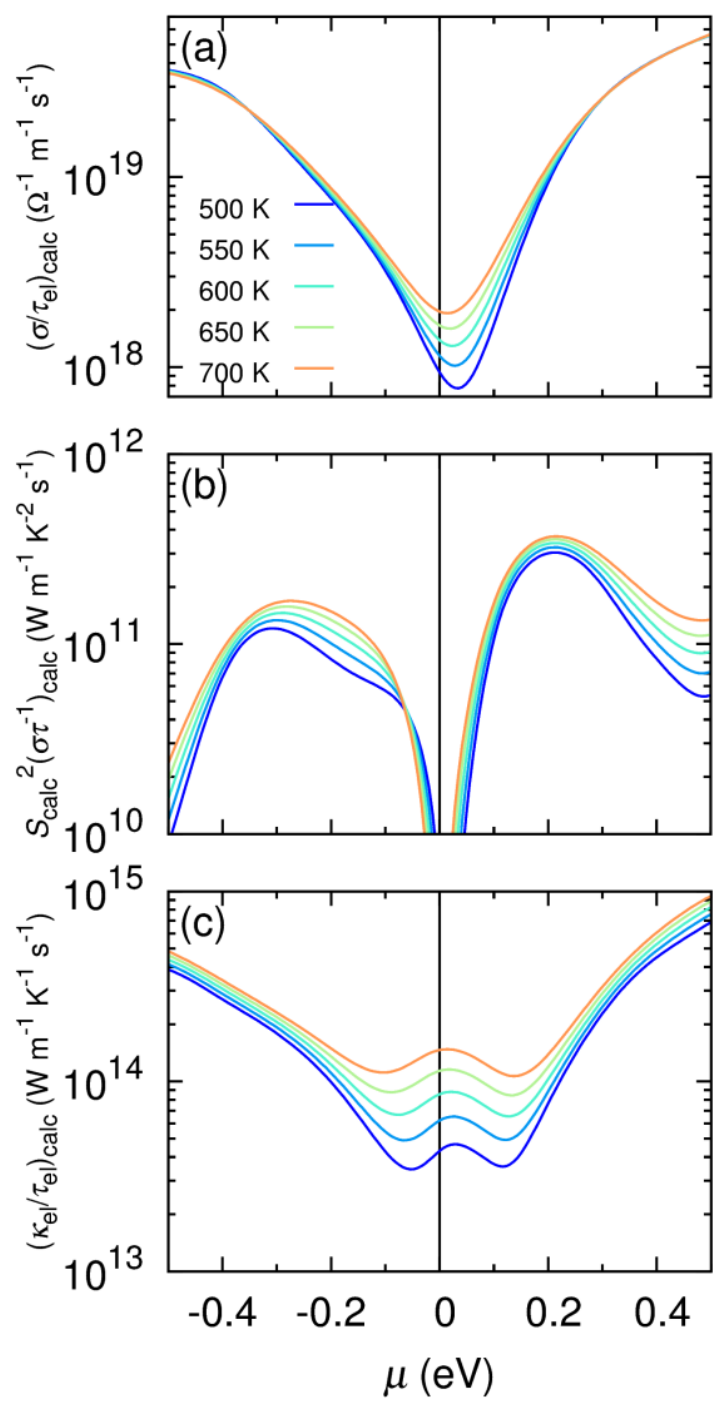

Figure S1. (a) Calculated electrical conductivity, $\left(\sigma / \tau_{\mathrm{el}}\right)_{\text {calc }}$, (b) power factor, $S_{\text {calc }}^{2}\left(\sigma / \tau_{\mathrm{el}}\right)_{\text {calc }}$, and (c) electron thermal conductivity, $\left(\kappa_{\mathrm{el}} / \tau_{\mathrm{el}}\right)_{\text {calc }}$, respectively, for hypothetical $\mathrm{Fe}_{2} \mathrm{Ge}_{3}$ from $500 \mathrm{~K}$ to $700 \mathrm{~K}$ as a function of the chemical potential, $\mu$. 


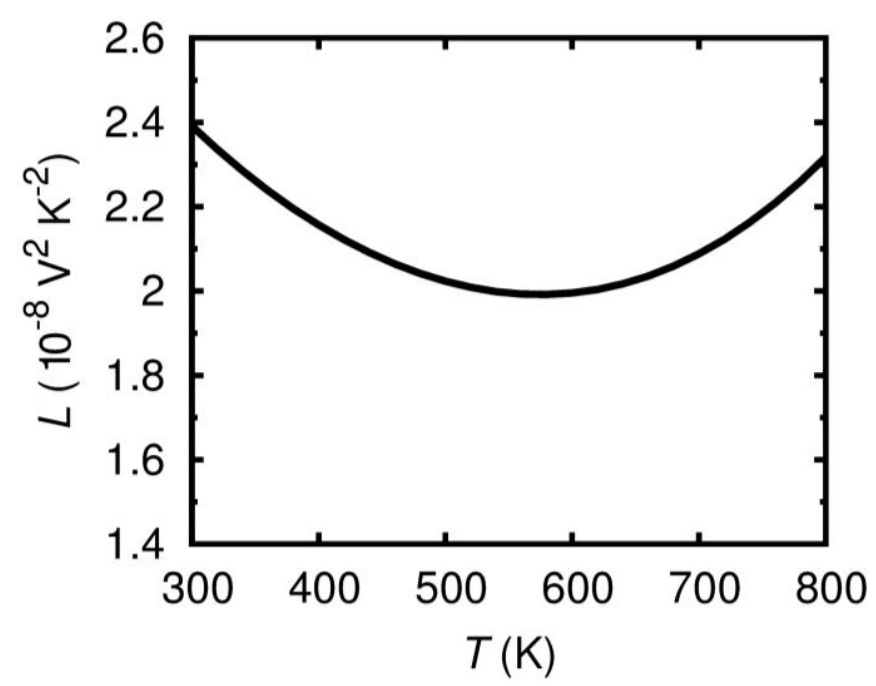

Figure S2. Temperature dependence of Lorenz number, $L$, estimated by using calculated electrical conductivity and electron thermal conductivity for hypothetical $\mathrm{Fe}_{2} \mathrm{Ge}_{3}$. 


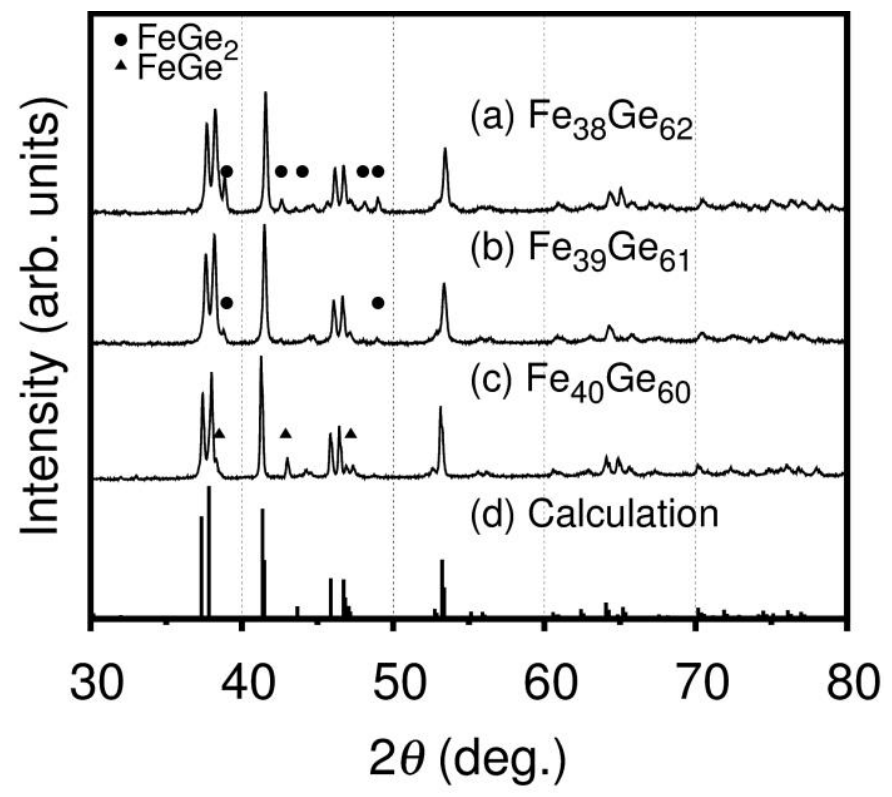

Figure S3. (a-c) X-ray diffraction patterns for sintered bulk $\mathrm{Fe}_{38+x} \mathrm{Ge}_{62-x}(x=0,1$, and 2), together with calculated pattern for the hypothetical $\mathrm{Ru}_{2} \mathrm{Sn}_{3}$-type $\mathrm{Fe}_{2} \mathrm{Ge}_{3}$ (d). The filled circle and triangle indicate peaks of secondary phases of $\mathrm{FeGe}_{2}$ and $\mathrm{FeGe}$, respectively. 